SOI: $1.1 /$ TAS DOI: $10.15863 /$ TAS

International Scientific Journal Theoretical \& Applied Science

p-ISSN: 2308-4944 (print) $\quad$ e-ISSN: 2409-0085 (online)

Year: $2016 \quad$ Issue: 1 Volume: 33

Published: $30.01 .2016 \quad \underline{\text { http://T-Science.org }}$
Alexander Danilovich Chernyshov Doctor of physico-mathematical Sciences, professor The Voronezh State University of Engineering Technology, Russia. chernyshovad@mail.ru

Vitalij Valer'evich Goryajnov Candidate of physico-mathematical Sciences, associate prof. The Voronezh State University of Architecture and Civil Engineering, Russia. gorvit77@mail.ru

SECTION 2. Applied mathematics. Mathematical modeling.

\title{
ON THE RELATIONSHIP BETWEEN THE NUMBER OF POINTS OF INFLECTION OF THE GRAPH OF THE NUMBER OF MEMBERS FOURIER SERIES BY USING RAPID EXPANSIONS
}

Abstract: We studied the relative error of representation functions and their derivatives rapid expansions of sine and cosine expansion. Based on the analysis of data obtained by the condition of numerical experiments to determine the number of members of the rapid expansion required to represent smooth functions rapid expansion with relative error calculation is not more than $1 \%$.

Key words: Rapid expansions, computing experiment, an error, inflection point.

Language: Russian

Citation: Chernyshov AD, Goryajnov VV (2016) ON THE RELATIONSHIP BETWEEN THE NUMBER OF POINTS OF INFLECTION OF THE GRAPH OF THE NUMBER OF MEMBERS FOURIER SERIES BY USING RAPID EXPANSIONS. ISJ Theoretical \& Applied Science, 01 (33): 137-141.

Soi: http://s-o-i.org/1.1/TAS-01-33-23 Doi: crossef http://dx.doi.org/10.15863/TAS.2016.01.33.23

\section{О СВЯЗИ КОЛИЧЕСТВА ТОЧЕК ПЕРЕГИБА ГРАФИКА ФУНКЦИИ И КОЛИЧЕСТВА ЧЛЕНОВ РЯДОВ ФУРЬЕ ПРИ ИСПОЛЬЗОВАНИИ БЫСТРЫХ РАЗЛОЖЕНИЙ}

Аннотация: Исследована относительная погрешность представления функций и их производных быстрыми синус- и косинус-разложениями. На основе анализа данных вычислительных экспериментов получено условие для определения количества членов быстрого разложения необходимых для представления гладких функиий быстрым разложением с относительной погрешностью вычислений не более $1 \%$.

Ключевые слова: быстрые разложения, вычислительный эксперимент, погрешность, точки перегиба.

\section{Введение.}

Разработке и исследованию быстрых разложений посвящены работы [1-21]. В статьях $[5,6,8-11,15,18-21]$ для вычислительных экспериментов использовались функции, имеющие точки перегиба. Из этих работ, а также примеров, приведенных в [22] видно, что наличие точек перегиба у графиков гладких функций создает вычислительные трудности и существенно влияет на погрешность. В работе [10] было проведено исследование влияния количества точек перегиба на точность представления функции частичной суммой в быстром разложении. В [10] использован поточечный метод $[5-11,15,18-21]$ для нахождения коэффициентов Фурье и применялись только быстрые синус-разложения. В данной работе будет сформулировано условие, позволяющее определить количество членов быстрого разложения, необходимое для представления функции с точками перегиба и относительной погрешностью вычислений не более $1 \%$. При этом исследуемые функции будут представляться быстрыми синус- и косинусразложениями.

Вычислительные эксперименты и обсуждение результатов.

В качестве исследуемых функций с наличием точек перегиба используем

$$
\begin{aligned}
& f_{1}(x)=\sin (k+0.2) \pi x, x \in[0 ; 1], k=1,5,10(1) \\
& \text { и } \\
& f_{2}(x)=\cos (k+0.2) \pi x, x \in[0 ; 1], k=1,5,10 .(2) \\
& \text { Зависимости (1) и (2) на отрезке }[0,1] \text { имеют } \\
& k \text { точек перегиба. Число } 0.2 \text { выбрано }
\end{aligned}
$$$$
\text { и }
$$ 


\begin{tabular}{|c|c|c|c|c|c|c|}
\hline Impact Factor: & $\begin{array}{l}\text { ISRA (India) } \\
\text { ISI (Dubai, UAF } \\
\text { GIF (Australia) } \\
\text { JIF }\end{array}$ & $\begin{array}{l}=1.344 \\
=0.829 \\
=0.564 \\
=1.500\end{array}$ & $\begin{array}{l}\text { SIS (USA) } \\
\text { PИНЦ (Russia) } \\
\text { ESJI (KZ) } \\
\text { SJIF (Morocco) }\end{array}$ & $\begin{array}{l}=0.912 \\
=0.179 \\
=1.042 \\
=2.031\end{array}$ & $\begin{array}{l}\text { ICV (Poland) } \\
\text { PIF (India) }\end{array}$ & $\begin{array}{l}=6.630 \\
=1.940\end{array}$ \\
\hline
\end{tabular}

специально для того, чтобы при $x=1$ значения аргумента не были кратными $\pi$, что создает дополнительные вычислительные трудности и увеличивает погрешность.

Функции (1) и (2) представим как быстрыми синус-, так и косинус-разложениями, которые являются суммами граничной функции специального вида и ряда Фурье по синусам или косинусам соответственно

$$
\begin{aligned}
& f(x)=M_{2 p}(x)+\sum_{m=1}^{\infty} b_{m}^{*} \sin m \pi x, \\
& b_{m}^{*}=2 \int_{0}^{1}\left(f(x)-M_{2 p}(x)\right) \sin m \pi x d x, \\
& \left(f(x), M_{2 p}(x)\right) \in C^{(2 p+1)}(x \in[0,1]), \\
& \left(f(x), M_{2 p}(x)\right) \in C^{(2 p+2)}(x \in(0,1)) \\
& f(x)=M_{2 p-1}(x)+a_{0}^{*}+\sum_{m=1}^{\infty} a_{m}^{*} \cos m \pi x, \\
& a_{0}^{*}=2 \int_{0}^{1}\left(f(x)-M_{2 p-1}(x)\right) d x, \\
& a_{m}^{*}=2 \int_{0}^{1}\left(f(x)-M_{2 p-1}(x)\right) \cos m \pi x d x, \\
& \left(f(x), M_{2 p-1}(x)\right) \in C^{(2 p)}(x \in[0,1]), \\
& \left(f(x), M_{2 p-1}(x)\right) \in C^{(2 p+1)}(x \in(0,1)),
\end{aligned}
$$

где $a_{0}^{*}, a_{m}^{*}, b_{m}^{*}$ - коэффициенты Фурье, $M_{2 p}(x)$ и $M_{2 p-1}(x)$ - граничные функции, которые имеют вид $[1,17]$

$$
\begin{gathered}
M_{2 p}(x)=f(0)(1-x)+f(1) x+ \\
+\sum_{i=1}^{p} f^{(2 i)}(0) A_{2 i}(x)+ \\
+\sum_{i=1}^{p} f^{(2 i)}(1) B_{2 i}(x), \quad p \geq 0, \\
M_{2 p-1}(x)=\sum_{i=1}^{p} f^{(2 i-1)}(0) A_{2 i-1}(x)+ \\
+\sum_{i=1}^{p} f^{(2 i-1)}(1) B_{2 i-1}(x), \quad p \geq 1,
\end{gathered}
$$

$$
\begin{aligned}
& A_{2 i}(x)=\Phi_{2 i}(x)-x \Phi_{2 i}(1), \\
& \Phi_{2 i}(x)=\int_{0}^{x}\left[\int_{0}^{x} A_{2 i-2}(x) d x\right] d x, \\
& A_{0}(x)=1-x, B_{0}(x)=x, \\
& B_{2 i}(x)=F_{2 i}(x)-x F_{2 i}(1), \\
& F_{2 i}(x)=\int_{0}^{x}\left[\int_{0}^{x} B_{2 i-2}(x) d x\right] d x, \\
& A_{2 i-1}(x)=\int_{0}^{x} A_{2 i-2}(x) d x \\
& B_{2 i-1}(x)=\int_{0}^{x} B_{2 i-2}(x) d x .
\end{aligned}
$$

В разложении (3) использовались граничные функции $M_{2}(x), M_{4}(x), M_{6}(x)$, а в (4) $M_{1}(x), M_{3}(x), M_{5}(x)$. В рядах Фурье ограничимся $n$ членами.

Во внутренних точках отрезка $[0,1]$

\begin{tabular}{|c|c|c|c|c|c|c|c|c|c|c|c|c|}
\hline \multirow{3}{*}{ Функция } & \multicolumn{12}{|c|}{ Граничная функция } \\
\hline & \multicolumn{2}{|c|}{$M_{1}$} & \multicolumn{2}{|c|}{$M_{2}$} & \multicolumn{2}{|c|}{$M_{3}$} & \multicolumn{2}{|r|}{$M_{4}$} & \multicolumn{2}{|r|}{$M_{5}$} & \multicolumn{2}{|r|}{$M_{6}$} \\
\hline & $n$ & $\begin{array}{c}\delta_{\max } \\
\%\end{array}$ & $n$ & $\begin{array}{c}\delta_{\max }, \\
\%\end{array}$ & $n$ & $\begin{array}{c}\delta_{\max }, \\
\%\end{array}$ & $n$ & $\begin{array}{c}\delta_{\max } \\
\%\end{array}$ & $n$ & $\delta_{\max }, \%$ & $n$ & $\delta_{\max }, \%$ \\
\hline $\sin 1.2 \pi x$ & 4 & 0,31 & 2 & 0.47 & 2 & 0,14 & 2 & $6,8410^{-2}$ & 2 & $1,5210^{-2}$ & 1 & 0.50 \\
\hline $\sin 5.2 \pi x$ & 14 & 0,97 & 9 & 0.96 & 9 & 0,96 & 8 & 0.43 & 8 & 0,26 & 7 & 0.38 \\
\hline $\sin 10.2 \pi x$ & 29 & 0,90 & 18 & 1,00 & 17 & 0,99 & 15 & 0.73 & 15 & 0,58 & 13 & 0.90 \\
\hline $\cos 1.2 \pi x$ & 3 & 0,52 & 2 & 0,40 & 2 & 0,19 & 2 & $4,2810^{-2}$ & 1 & 0,85 & 2 & $5,1510^{-3}$ \\
\hline $\cos 5.2 \pi x$ & 12 & 1,00 & 10 & 0,87 & 8 & 0,86 & 8 & 0,51 & 7 & 0,63 & 7 & 0,91 \\
\hline
\end{tabular}
относительная погрешность функций (1), (2) и их производных до $(2 p+1)$ порядка включительно исследовалась по формуле

$$
\delta=|\Delta| / N \cdot 100 \%,
$$

где $\Delta-$ абсолютная погрешность функции (производной), $N-$ максимальное значение функции (производной).

Результаты вычислительных экспериментов приведены в табл. 1 - 3. Из табл. 1 - 3 видно, что быстрым синус-разложением точнее описываются функции (1), чем функции (2), а в случае использования быстрого косинусразложения точнее описываются функции (2), чем (1). Это выражается в меньшей погрешности $\delta_{\max }(f)$ при одинаковом количестве членов $n$ в рядах Фурье быстрых разложений. Подобная ситуация становится заметнее с увеличением количества точек перегиба графика функций (1) и (2) и увеличением порядка их производных.

Количество расчетных точек, необходимых для $\delta_{\max }(f)<1 \%$. 


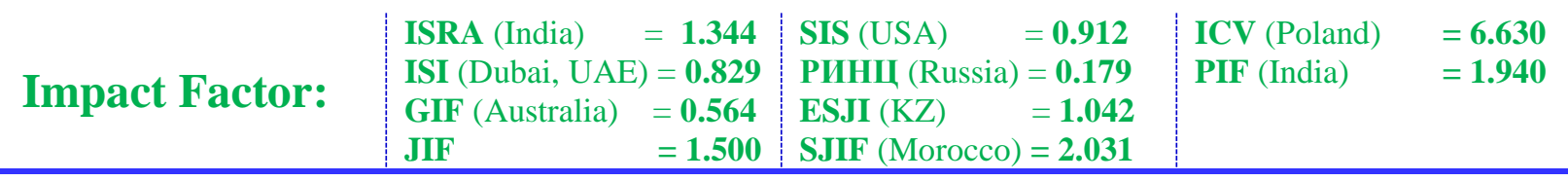

\begin{tabular}{|l|l|l|l|l|l|l|l|l|l|l|l|l|}
\hline $\cos 10.2 \pi x$ & 25 & 0,88 & 21 & 0,80 & 16 & 0,93 & 16 & 0,93 & 14 & 0,74 & 14 & 0,96 \\
\hline
\end{tabular}

\section{Таблица 2}

Количество расчетных точек, необходимых для $\delta_{\max }\left(f^{\prime}\right)<1 \%$.

\begin{tabular}{|c|c|c|c|c|c|c|c|c|c|c|c|c|}
\hline \multirow{3}{*}{$\begin{array}{c}\text { Первая } \\
\text { производна } \\
\text { я функции }\end{array}$} & \multicolumn{12}{|c|}{ Граничная функция } \\
\hline & \multicolumn{2}{|c|}{$M_{1}$} & \multicolumn{2}{|c|}{$M_{2}$} & \multicolumn{2}{|c|}{$M_{3}$} & \multicolumn{2}{|c|}{$M_{4}$} & \multicolumn{2}{|r|}{$M_{5}$} & \multicolumn{2}{|r|}{$M_{6}$} \\
\hline & $n$ & $\begin{array}{c}\delta_{\max }, \\
\%\end{array}$ & $n$ & $\begin{array}{l}\delta_{\max } \\
, \% \\
\end{array}$ & $n$ & $\begin{array}{c}\delta_{\max }, \\
\%\end{array}$ & $n$ & $\begin{array}{c}\delta_{\max }, \\
\%\end{array}$ & $n$ & $\delta_{\max }, \%$ & $n$ & $\delta_{\max }, \%$ \\
\hline $\sin 1.2 \pi x$ & 6 & 0,74 & 3 & 0,52 & 2 & 0,40 & 2 & 0,19 & 2 & $4,8210^{-2}$ & 1 & 0,85 \\
\hline $\sin 5.2 \pi x$ & 26 & 0,93 & 12 & 1,00 & 10 & 0,87 & 8 & 0,86 & 8 & 0,51 & 7 & 0,63 \\
\hline $\sin 10.2 \pi x$ & 51 & 0,96 & 25 & 0,88 & 21 & 0,80 & 16 & 0,93 & 16 & 0,93 & 14 & 0,74 \\
\hline $\cos 1.2 \pi x$ & 5 & 0,70 & 4 & 0,31 & 2 & 0,47 & 2 & 0,14 & 2 & $6,8410^{-2}$ & 2 & $1,5210^{-2}$ \\
\hline $\cos 5.2 \pi x$ & 20 & 0,96 & 14 & 0,97 & 9 & 0,71 & 9 & 0,96 & 8 & 0,43 & 8 & 0,26 \\
\hline $\cos 10.2 \pi x$ & 39 & 0,99 & 29 & 0,90 & 18 & 1,00 & 17 & 0,99 & 15 & 0,73 & 15 & 0,58 \\
\hline
\end{tabular}

Таблица 3

Количество расчетных точек, необходимых для $\delta_{\max }\left(f^{\prime \prime}\right)<1 \%$.

\begin{tabular}{|c|c|c|c|c|c|c|c|c|c|c|c|c|}
\hline \multirow{3}{*}{$\begin{array}{c}\text { Вторая } \\
\text { производна } \\
\text { я функции }\end{array}$} & \multicolumn{12}{|c|}{ Граничная функция } \\
\hline & \multicolumn{2}{|c|}{$M_{1}$} & \multicolumn{2}{|c|}{$M_{2}$} & \multicolumn{2}{|c|}{$M_{3}$} & \multicolumn{2}{|c|}{$M_{4}$} & \multicolumn{2}{|c|}{$M_{5}$} & \multicolumn{2}{|r|}{$M_{6}$} \\
\hline & $n$ & $\begin{array}{c}\delta_{\max } \\
\%\end{array}$ & $n$ & $\begin{array}{c}\delta_{\max } \\
, \%\end{array}$ & $n$ & $\begin{array}{c}\delta_{\max } \\
\%\end{array}$ & $n$ & $\begin{array}{c}\delta_{\max }, \\
\%\end{array}$ & $n$ & $\delta_{\max }, \%$ & $n$ & $\delta_{\max }, \%$ \\
\hline $\sin 1.2 \pi x$ & 76 & 0,99 & 5 & 0,70 & 4 & 0,31 & 2 & 0,47 & 2 & 0,14 & 2 & $6,8410^{-2}$ \\
\hline $\sin 5.2 \pi x$ & 330 & 1,00 & 20 & 0,96 & 14 & 0,97 & 9 & 0,96 & 9 & 0,96 & 8 & 0,43 \\
\hline $\sin 10.2 \pi x$ & 649 & 1,00 & 39 & 0,99 & 29 & 0,90 & 18 & 1,00 & 17 & 0,99 & 15 & 0,73 \\
\hline $\cos 1.2 \pi x$ & 45 & 0,99 & 6 & 0,74 & 3 & 0,52 & 2 & 0,40 & 2 & 0,19 & 2 & $4,2810^{-2}$ \\
\hline $\cos 5.2 \pi x$ & 195 & 1,00 & 26 & 0,93 & 12 & 1,00 & 10 & 0,87 & 8 & 0,86 & 8 & 0,51 \\
\hline $\cos 10.2 \pi x$ & 381 & 1.00 & 51 & 0,96 & 25 & 0,88 & 21 & 0,80 & 16 & 0,93 & 16 & 0,93 \\
\hline
\end{tabular}

\section{Выводы.}

Если функция имеет $k$ точек перегиба, то в ряде Фурье должно быть членов больше $k$, т.е. должно выполняться условие

$$
n>k \text {. }
$$

Следует отметить, что условие (5) необходимо выполнять при использовании в быстром разложении граничных функций различного порядка.

По исследуемым функциям (1) и (2) можно сделать вывод, что для обеспечения погрешности $\delta_{\max }(f)<1 \%$ в рядах Фурье необходимо учитывать не менее $2 k$ членов, для $\delta_{\max }\left(f^{\prime}\right)<1 \%$ - не менее $3 k$ членов, а для $\delta_{\max }\left(f^{\prime \prime}\right)<1 \%$ - не менее $4 k$ членов (при использовании граничной функции порядка не менее второго, т.е $\left.M_{2}(x)\right)$.

Если $k=0$ и кривая, описываемая быстрым разложением, представляет собой функцию, не имеющую особенности быстро убывать и прижиматься к оси $x$, то, учитывая данные [10], можно сделать вывод, что в этом случае достаточно одного члена ряда Фурье при использовании граничной функции порядка не менее второго, т.е $M_{2}(x)$.

\section{References:}

1. Chernyshov AD (2009) Uluchshennye ryady Fur'e i granichnye funktsii // Aktual'nye problemy prikladnoj matematiki, informatiki i mekhaniki / Sb. tr. mezhdunar. konf. Voronezh.: VGU, 2009, Ch. 2. - pp. $236-238$.
2. Chernyshov AD (2010) Bystrye ryady Fur'e // Aktual'nye problemy prikladnoj matematiki, informatiki i mekhaniki / Sb. tr. mezhdunar. konf. - Voronezh.: VGU, 2010. pp. 388 - 394. 


\begin{tabular}{|c|c|c|c|c|c|c|}
\hline Impact Factor: & $\begin{array}{l}\text { ISRA (India) } \\
\text { ISI (Dubai, UAE } \\
\text { GIF (Australia) } \\
\text { JIF }\end{array}$ & $\begin{array}{r}=1.344 \\
=0.829 \\
=0.564 \\
=1.500\end{array}$ & $\begin{array}{l}\text { SIS (USA) } \\
\text { PUHЦ (Russia } \\
\text { ESJI (KZ) } \\
\text { SJIF (Morocce }\end{array}$ & $\begin{array}{l}=0.912 \\
=0.179 \\
=1.042 \\
=2.031\end{array}$ & $\begin{array}{l}\text { ICV (Poland) } \\
\text { PIF (India) }\end{array}$ & $\begin{array}{l}=6.630 \\
=1.940\end{array}$ \\
\hline
\end{tabular}

3. Chernyshov AD (2010) Uluchshenie differentsiruemosti reshenij kraevyh zadach mekhaniki $\mathrm{v}$ forme obobshchennyh ryadov Fur'e s pomoshch'yu granichnyh funktsij. Izv. RAN. Mekhan. tv. tela. 2010. № 1, pp. 174192.

4. Chernyshov AD (2010) Postroenie dvumernoj granichnoj funktsii dlya bystryh ryadov Fur'e. Vestnik CHuvashskogo gosudarstvennogo pedagogicheskogo universiteta im. I.YA. YAkovleva. Seriya: Mekhanika predel'nogo sostoyaniya - № 8. - 2010. - pp. 535-540.

5. Chernyshov AD, Goryajnov VV, Solov'ev AO (2010) O vozmozhnosti vychisleniya koehffitsientov Fur'e potochechnym metodom // Vestnik Voronezhskogo gosudarstvennogo tekhnicheskogo universiteta. - T. 6. - № 2. 2010. - pp. $49-53$.

6. Goryajnov VV (2010) Ustojchivost' potochechnogo metoda vychisleniya koehffitsientov bystryh ryadov Fur'e // Aktual'nye problemy prikladnoj matematiki, informatiki i mekhaniki / Sb. tr. mezhdunar. konf. - Voronezh.: VGU, 2010. pp. 120 - 124.

7. Chernyshov AD (2011) O primenenii bystryh razlozhenij dlya resheniya nelinejnyh zadach mekhaniki // Aktual'nye problemy prikladnoj matematiki, informatiki i mekhaniki / Sb. tr. mezhdunar. konf. - Voronezh.: VGU, 2011. pp. $412-416$.

8. Goryajnov VV (2011) Analiz pogreshnosti bystryh ryadov Fur'e pri ih mnogokratnom differentsirovanii dlya sluchaya vychisleniya koehffitsientov ryada potochechnym metodom // Vestnik Voronezhskogo gosudarstvennogo tekhnicheskogo universiteta. T. 7. № 2. 2011. pp. $36-40$.

9. Chernyshov AD, Hozyainova NA, Goryajnov VV (2011) Issledovanie pogreshnosti potochechnogo metoda vychisleniya koehffitsientov bystryh ryadov Fur'e // Vestnik Voronezhskoj gosudarstvennoj tekhnologicheskoj akademii. Ser. Informatsionnye tekhnologii, modelirovanie i upravlenie. № 2. 2011. pp. $64-67$.

10. Chernyshov AD, Goryainov VV (2011) O vybore optimal'nogo poryadka granichnoj funktsii $\mathrm{v}$ bystrom razlozhenii // Vestnik Voronezhskogo gosudarstvennogo universiteta. Seriya: Sistemnyj analiz i informatsionnye tekhnologii. 2011. №1. pp. $60-65$.

11. Chernyshov AD, Goryajnov VV (2011) O sravnenii bystryh sinus - i kosinus-razlozhenij v kraevyh zadachah s usloviyami Dirihle // Aktual'nye problemy prikladnoj matematiki, informatiki i mekhaniki / Sb. tr. mezhdunar. konf. - Voronezh.: VGU, 2011. pp. 417 - 422.

12. Chernyshov AD (2012) Operator bystryh razlozhenij i teorema edinstvennosti bystryh razlozhenij // Aktual'nye problemy prikladnoj matematiki, informatiki i mekhaniki / Sb. tr. mezhdunar. konf. - Voronezh.: VGU, 2012, ch. 1. pp. $401-405$.

13. Chernyshov AD, Pavlov IO, Voronova EV, Goryajnov VV (2012) Reshenie metodom bystryh razlozhenij zadachi o sushke zerna // Teplofizika i aehromekhanika, 2012, tom 19, № 6. pp. 739-749.

14. Chernyshov AD, Goryajnov VV (2012) Reshenie odnogo nelinejnogo integrodifferentsial'nogo uravneniya metodom bystryh razlozhenij // Vestnik CHGPU im. I.YA. YAkovleva. Seriya: mekhanika predel'nogo sostoyaniya. № 4(12). 2012. pp. 105 - 112 .

15. Chernyshov AD, Goryajnov VV (2012) O sposobe naneseniya raschetnyh tochek na otrezok pri realizatsii potochechnogo metoda vychisleniya koehffitsientov bystryh razlozhenij dlya resheniya kraevoj zadachi s usloviyami Dirihle // Vestnik Voronezhskogo gosudarstvennogo universiteta. Seriya: Sistemnyj analiz i informatsionnye tekhnologii. № 2. 2012. pp. $30-35$.

16. Chernyshov AD, Goryajnov VV (2012) Ob uluchshenii skhodimosti bystryh razlozhenij za schet vybora vida granichnoj funktsii // Aktual'nye problemy prikladnoj matematiki, informatiki i mekhaniki / Sb. tr. mezhdunar. konf. - Voronezh.: VGU, 2012. pp. 406 - 409.

17. Chernyshov AD (2014) Metod bystryh razlozhenij dlya resheniya nelinejnyh differentsial'nyh uravnenij // Zhurnal Vychislitel'noi Matematiki i Matematicheskoi Fiziki, 2014, vol. 54, no. 1, pp. 13-24.

18. Chernyshov AD, Goryajnov VV, Shahov AS (2014) Programma dlya EHVM «Predstavlenie funktsii iz klassa $C^{(m)}$ ( $m$ - konechnoe chislo) bystrym sinus razlozheniem, $\mathrm{v}$ kotorom ispol'zovana granichnaya funktsiya vtorogo poryadka, a koehffitsienty razlozheniya najdeny po-tochechnym metodom pri ravnomernom razbienii otrezka» №2014617687 // Programmy dlya EHVM. Bazy dannyh. Topologii integral'nyh mikroskhem, № 8(94), 2014.

19. Chernyshov AD, Goryajnov VV, Shahov SV (2014) Programma dlya EHVM «Predstavlenie funktsii iz klassa $C^{(m)}(m-$ konechnoe chislo) bystrym kosinus razlozheniem, $\mathrm{v}$ kotorom ispol'zovana granichnaya funktsiya tret'ego poryadka, a koehffitsienty razlozheniya najdeny potochechnym metodom pri ravnomernom razbienii otrezka» №2014662206 // Programmy dlya EHVM. Bazy dannyh. Topologii integral'nyh mikroskhem, № 12(98), 2014.

20. Chernyshov AD, Goryajnov VV, Shahov SV () Programma dlya EHVM «Predstavlenie funktsii iz klassa $C^{(m)}$ ( $m$ - konechnoe chislo) bystrym 


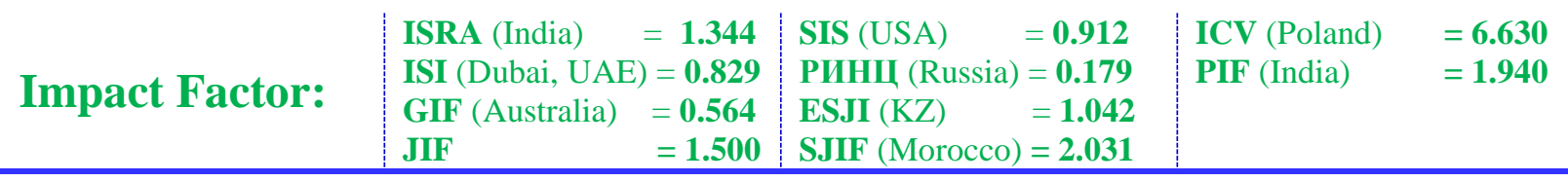

sinus razlozheniem, v kotorom ispol'zovana granichnaya funktsiya chetvertogo poryadka, a koehffitsienty razlozheniya najdeny potochechnym metodom pri ravnomernom razbienii otrezka» №2015614809 // Programmy dlya EHVM. Bazy dannyh. Topologii integral'nyh mikroskhem, № 5(103), 2015.

21. Chernyshov AD, Goryajnov VV, Shahov SV (2015) Programma dlya EHVM «Predstavlenie funktsii iz klassa $C^{(m)}$ ( $m$ - konechnoe chislo) bystrym kosinus razlozheniem, $\mathrm{v}$ kotorom ispol'zovana granichnaya funktsiya pyatogo poryadka, a koehffitsienty razlozheniya najdeny potochechnym meto-dom pri ravnomernom razbienii otrezka» №2015615081 // Programmy dlya EHVM. Bazy dannyh. Topologii integral'nyh mikroskhem, № 6(104), 2015.

22. Bahvalov NS, Zhidkov NP, Kobel'kov GM (1987) Chislennye metody. - Moscow: Nauka, 1987. $-600 \mathrm{p}$. 